\title{
Salivary amylase activity is useful for assessing perioperative stress in response to pain in patients undergoing endoscopic submucosal dissection of gastric tumors under deep sedation
}

\author{
Masaya Uesato $^{1}$, Yoshihiro Nabeya ${ }^{1}$, Takashi Akai $^{1}$, Masahito Inoue ${ }^{2}$, Yoshiyuki Watanabe ${ }^{2}$, \\ Hiroshi Kawahira ${ }^{1,3}$, Toshitaka Mamiya ${ }^{1}$, Yoshinito Ohta ${ }^{1}$, Ryuj Motojima $^{1}$, Akiko Kagaya ${ }^{1}$, Yorihiko Muto ${ }^{1}$, \\ Hideki Hayashi ${ }^{1,3}$, and Hisahiro Matsubara ${ }^{1}$ \\ ${ }^{1}$ Department of Frontier Surgery (M9), Chiba University Graduate School of Medicine, 1-8-1 Inohana, Chuo-ku, Chiba 260-8670, Japan \\ ${ }^{2}$ Department of Endoscopic Diagnostics and Therapeutics, Chiba University Hospital, Chiba, Japan \\ ${ }^{3}$ Research Center for Frontier Medical Engineering, Chiba University, Chiba, Japan
}

\begin{abstract}
Background. Although endoscopic submucosal dissection (ESD) for patients with gastric tumors under the conditions of unconsciousness is considered to be minimally invasive, no objective assessment of the perioperative stress of ESD has yet been conducted. Today, stress levels can be easily and objectively assessed by monitoring salivary amylase activity (SAMY). We evaluated the perioperative changes in the SAMY in patients undergoing ESD and identified the causes of such changes.

Methods. A total of 40 patients with gastric cancers/adenomas removed by ESD under general anesthesia (GA; $\boldsymbol{n}=20)$ and under deep sedation (DS; $n=20$ ) were enrolled. SAMY was measured using the enzyme analysis equipment, sAMY Monitor (NIPRO, Osaka, Japan) during the perioperative period of the ESD. Also, all patients were interviewed to determine their subjective stress level, using a questionnaire asking "How did you feel during ESD?", with the choice of responses ranging from "did not wake up at all" to "I was awake and ESD was extremely stressful".

Results. The sAMY of the DS group increased soon after the start of ESD. Meanwhile, that of the GA group decreased just after the ESD started and was maintained at a stable level throughout the ESD. In response to the stress level questionnaire, all of the patients in the GA group and a majority of the patients in the DS group responded, "did not wake up at all".

Conclusion. Sympathetic agitation, expressed as an increase of sAMY, was absent in the GA group. Meanwhile, in the DS group, some patients showed high levels of SAMY which went down following the administration of an analgesic agent, thus suggesting that pain caused an elevation in the level of the stress and thereby induced an increase in SAMY. The measurement of SAMY is therefore considered to be useful for the assessment of analgesic status under DS.
\end{abstract}

Key words Salivary amylase $\cdot$ Stress $\cdot$ Endoscopic submucosal dissection $\cdot$ Sedation $\cdot$ Analgesia

Offprint requests to: $\mathrm{M}$. Uesato

Received: July 21, 2009 / Accepted: December 25, 2009

\section{Introduction and background}

Endoscopic submucosal dissection (ESD) is widely used to treat mucosal gastric cancers, because an en-bloc resection of the lesions by ESD provides a detailed pathological assessment and a possible radical cure [1, 2]. However, technical problems and expansion of the eligibility criteria for ESD can also cause complications and can prolong surgical time [1, 3, 4]. Therefore, in order to perform ESD safely and satisfactorily, patients should be maintained under an appropriate anesthesia regimen or sedation during the entire period of the ESD.

Deep sedation (DS) is defined as a drug-induced depression of consciousness during which patients cannot be easily aroused [5]. On the other hand, general anesthesia (GA) is defined as a drug-induced loss of consciousness during which patients are not arousable, even by painful stimulation [5]. DS is more frequently used than GA for ESD [3]. While endoscopic treatments performed in apparently unconscious patients under DS were assumed to have little invasive impact, DS sometimes appears to be insufficient in patients undergoing ESD for an extended time $[1,3,4]$. However, no objective assessment of the perioperative stress for patients during ESD has so far been conducted.

Recent studies have demonstrated the efficacy of assessing psychological stress objectively by monitoring salivary amylase activity (sAMY) [6, 7], and an instrument using this method to assess stress with rapidity and low invasiveness has been marketed for practical use $[8$, 9]. Against this background, the objectives of this study were to investigate the differences in SAMY between DS and GA during ESD, to determine the efficacy of sAMY as a potential index of a patient's stress level during therapeutic endoscopy, and also to elucidate the causes of such stress. 


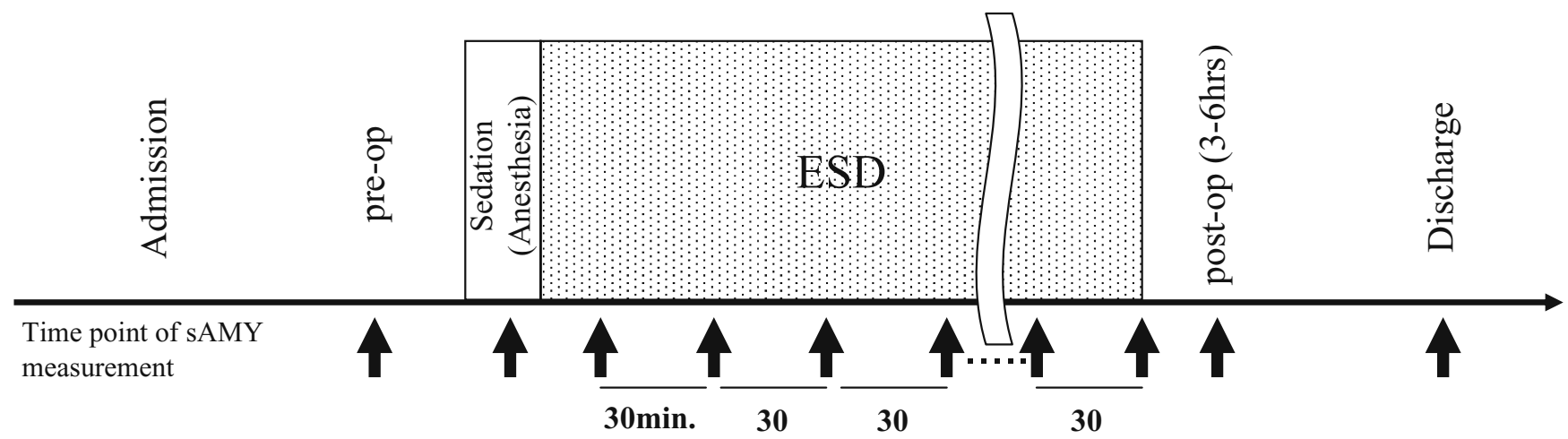

Fig. 1. Time points of salivary amylase activity ( $s A M Y)$ measurements (arrows). Pre-op, Preoperative; post-op, postoperative; $E S D$, endoscopic submucosal dissection

\section{Patients and methods}

This study enrolled 40 patients with early gastric cancer and/or adenoma who were treated at the Department of Frontier Surgery or the Department of Endoscopic Diagnostics and Therapeutics, Chiba University Hospital. Twenty patients were diagnosed by the attending endoscopists as having tumors (30 lesions) that might need a long time for en-bloc resection; therefore, they underwent ESD under GA, using propofol $(0.5 \mathrm{mg} /$ $\mathrm{kg} / 10 \mathrm{~min}$ i.v. $)$ and fentanyl (0.1 $\mathrm{mg}$ i.v.) maintained with sevoflurane, and were termed the GA group. The other 20 patients underwent ESD (for 22 lesions) under DS with midazolam $(0.04-0.06 \mathrm{mg} / \mathrm{kg}$ i.v. $)$ and pentazocine (7.5 mg i.v.) maintained properly, and were termed the DS group. Neither anticholinergic nor vasopressor agents were used.

sAMY was measured using the enzyme analysis equipment, sAMY Monitor (NIPRO, Osaka, Japan) prior to the ESD in the morning, just after the induction of sedation, every $30 \mathrm{~min}$ after the beginning of the ESD, 3 to $6 \mathrm{~h}$ after the end of the ESD, and just before discharge (Fig. 1). All patients were interviewed prior to discharge to determine their subjective stress level, using a questionnaire in regard to the question: "How did you feel during ESD?", and then they were asked to choose one of the following answers: (1)"I did not wake up at all", (2)"I was awake but ESD was not stressful", (3) "I was awake and ESD was slightly stressful", (4) "I was awake and ESD was mildly stressful", (5) "I was awake and ESD was moderately stressful", or (6) "I was awake and ESD was extremely stressful". In addition, a bispectral index monitor (BIS Monitor;Aspect Medical Systems, Norwood, MA, USA) was used to evaluate the level of consciousness.

The institutional review board approved the study protocol, and written informed consent was obtained from all patients.

\section{Statistical analysis}

Continuous data are presented as means \pm SD. The difference in sex proportions was evaluated using Fisher's exact test. The differences in age, body weight, longer axis of the dissected lesion, operation time, and perioperative changes in sAMY measurements between the groups were compared using the Mann-Whitney $U$-test. All calculations were done with SPSS 15.0 (SPSS, Chicago, IL, USA). $P$ values of less than 0.05 were considered to be statistically significant.

\section{Results}

Although the operation time was significantly longer in the GA group than in the DS group, no significant difference between the groups was found among the other patient characteristic factors (Table 1). Changes in the sAMY values in each group are shown in Fig. 2. Major changes in sAMY were observed over time in the DS group and minor changes were seen in the GA group. The sAMY level in the DS group increased just after the beginning of the ESD and it subsequently remained at higher levels during the operation. However, it did not continue to increase as time proceeded. Meanwhile, the sAMY value in the GA group decreased to a lower level just after the beginning of the operation and remained consistently low without any fluctuations thereafter. The mean sAMY values (in $\mathrm{kU} / \mathrm{l}$ ), with significant differences between the two groups (DS/GA: $P$ ) at 30,60, 90, and $150 \mathrm{~min}$ after the beginning of ESD, respectively, were: $(56.3 / 10.9$ : $<0.01)$, (68.5/8.9: <0.001), (51.0/7.9: <0.01), and (119.6/6.6: $<0.001)$. No significant differences between the two groups were observed regarding the preoperative findings, postoperative findings, and the findings just before discharge. 
Table 1. Characteristics of the patients in the groups undergoing DS or GA

\begin{tabular}{lccc}
\hline & DS group & GA group & $P$ value \\
\hline Number of patients & 20 & 20 & \\
Sex (male/female) & $12 / 8$ & $17 / 3$ & 0.18 \\
Age (years) & $68.1 \pm 7.6$ & $68.1 \pm 8.9$ & 0.66 \\
Body weight (kg) & $59.5 \pm 9.9$ & $62.6 \pm 9.6$ & 0.43 \\
Number of lesions & 22 & 30 & \\
$\quad$ Longer axis of dissected lesion (mm) & $34.0 \pm 14.1$ & $41.3 \pm 22.6$ & 0.53 \\
Operation time (min) & $120.5 \pm 83.2$ & $161.2 \pm 70.1$ & 0.038 \\
Range & $40-330$ & $60-295$ & \\
\hline
\end{tabular}

The operation time for the general anesthesia (GA) group was significantly longer than that for the deep sedation (DS) group

The data are presented as means \pm SD

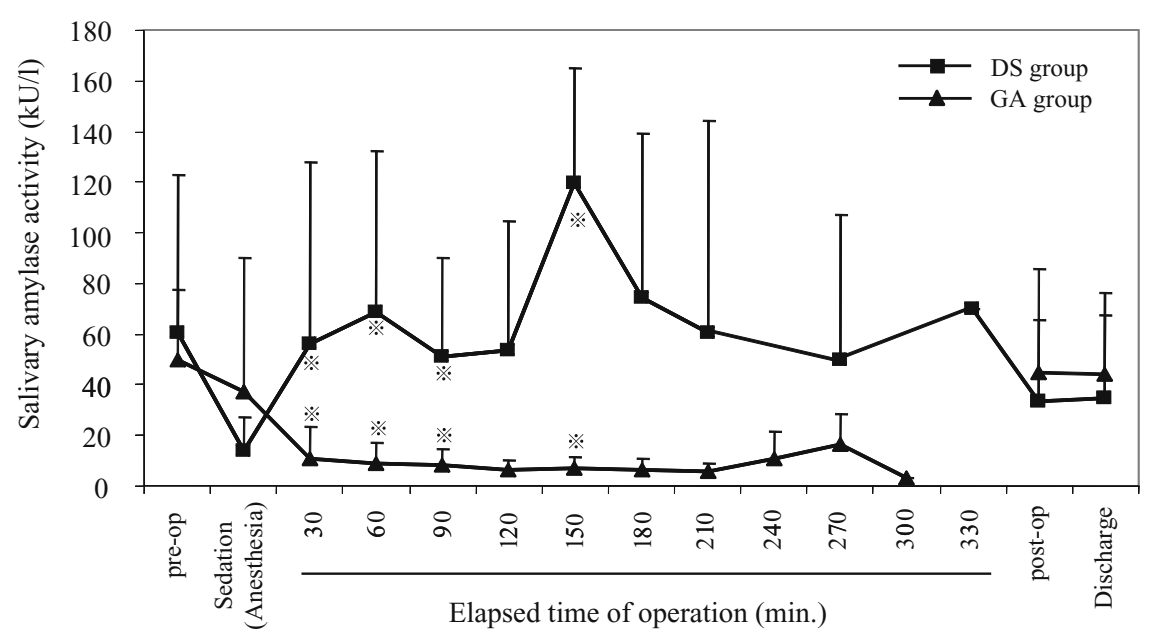

I did not wake up at all. I was awake and ESD was:
Fig. 2. Changes in the actual sAMY measurements in each group. Greater changes in sAMY were observed in the deep sedation $(D S)$ group and only slight changes were seen in the general anesthesia $(G A)$ group. Data are presented as means (bars, SD). Asterisks indicate significant differences $(P<0.05)$

Fig. 3. Results of the questionnaire. All of the patients in the GA group and a majority in the DS group did not subjectively experience any stress

The results of the questionnaire are shown in Fig. 3. All of the patients in the GA group responded, "did not wake up at all". A majority (12/20) of the DS group responded with either "did not wake up at all (8/20)",
"ESD was not stressful (3/20)", or "ESD was slightly stressful (1/20)". However, six subjects who gave the answer "I did not wake up at all" $(n=2)$, or "ESD was not stressful" $(n=3)$, or "ESD was only slightly stress- 
ful" $(n=1)$ did not necessarily receive ESD in a short time or demonstrate a low rate of sAMY changes in comparison to the baseline level just before discharge (Fig. 4).

The bispectral index in the DS group (78.3 $\pm 7.09 ; n$ $=7$ ) was significantly higher than that in the GA group $(50.9 \pm 10.8 ; n=16 ; P<0.001)$.

The clinical course of a patient in the DS group whose duration of ESD was $4 \mathrm{~h}$ is shown in Fig. 5. Little fluc- tuation of the bispectral index and no apparent awakening were observed. In contrast, body motion was observed at $30 \mathrm{~min}$ after the beginning of ESD, and midazolam was administered, but it failed to control the body motion, and a simultaneous increase occurred in sAMY. However, the administration of pentazocine was observed to successfully control the sAMY as well as the body motion. Similar results were observed in some other patients in the DS group.
I did not wake up at all. I was awake and ESD was:
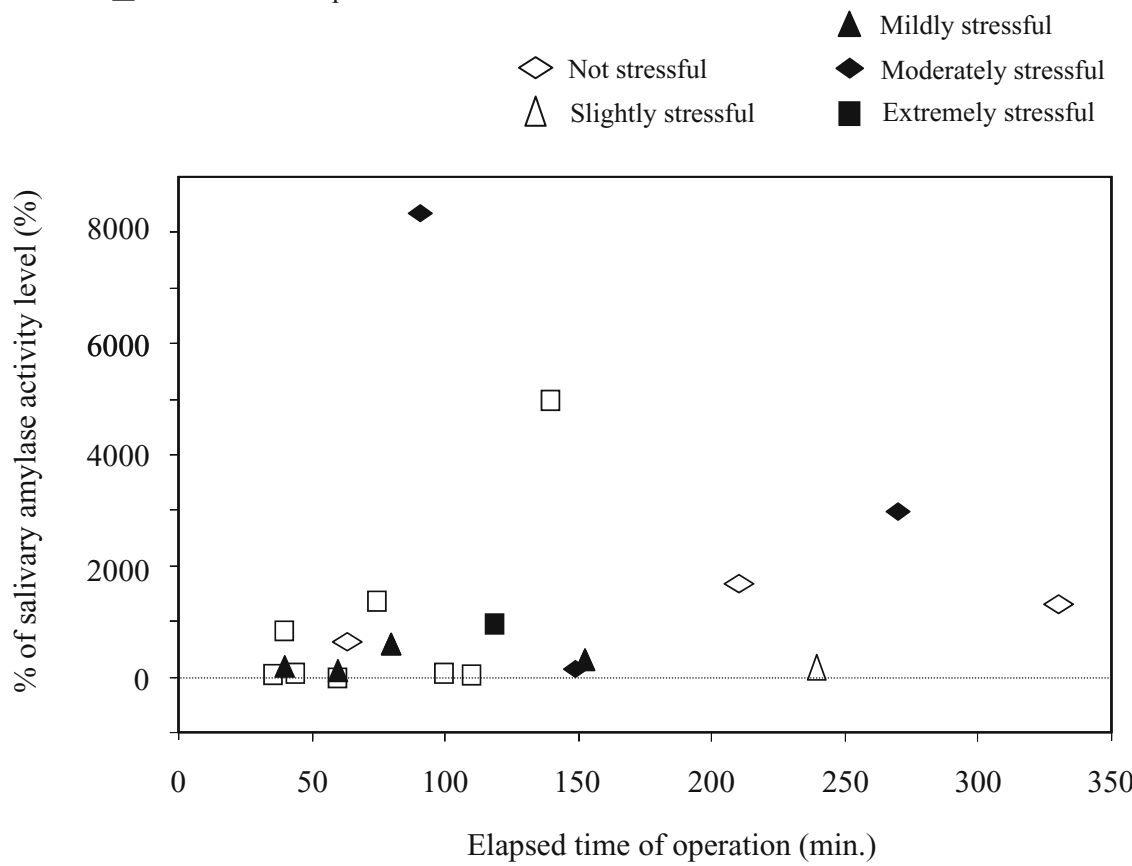

Fig. 4. The questionnaire results of each patient in the DS group are plotted. The horizontal axis shows the operation time, and the longitudinal axis shows the peak relative sAMY values, which are the percentage variations of the actual sAMY measurements in comparison to that before discharge. There was no correlation between the questionnaire results, the operation time, and the peak relative value of sAMY

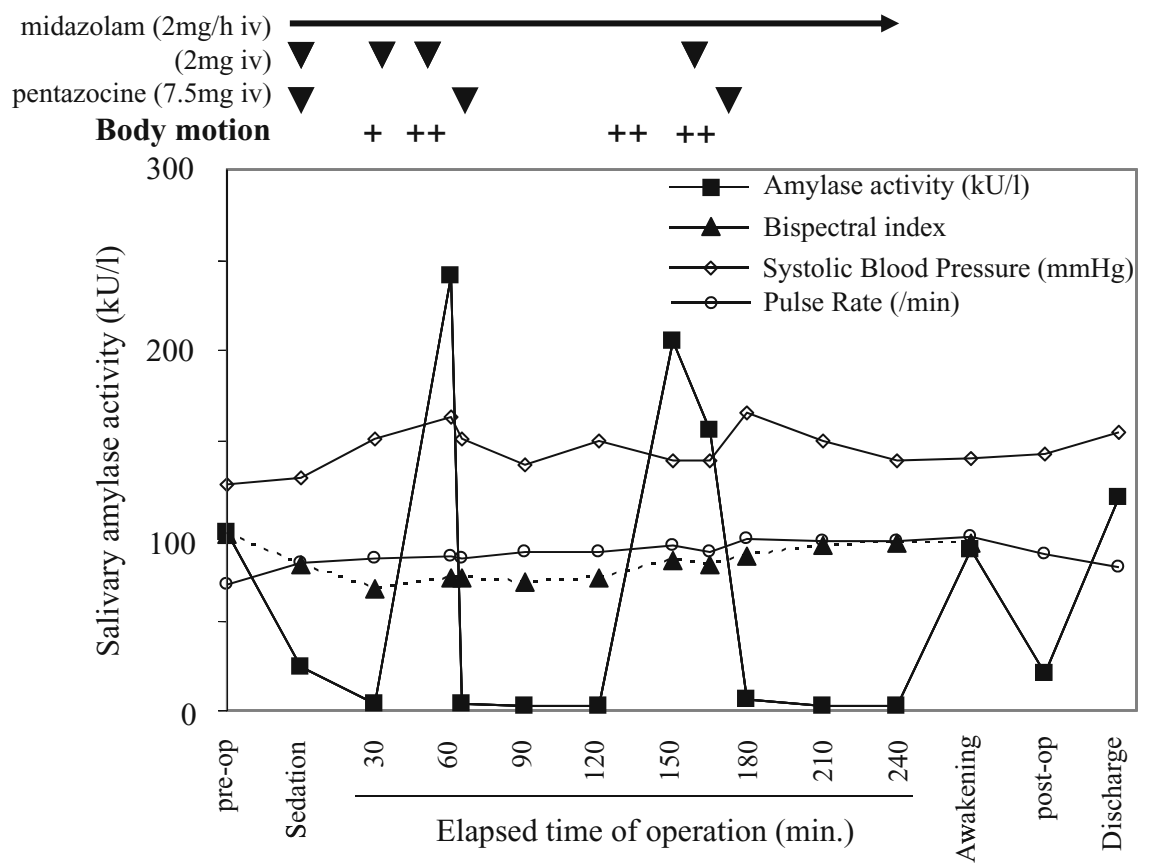

Fig. 5. The clinical course of a patient in the DS group whose duration of ESD was $4 \mathrm{~h}$. Midazolam $(2 \mathrm{mg} / \mathrm{h})$ was continuously infused during the operation (arrow). The time points of bolus injection of midazolam $(2 \mathrm{mg})$ and pentazocine $(7.5 \mathrm{mg})$ are indicated above the panel (arrowheads). The time points of observed body motion (+) are indicated below the arrowheads. Changes in the sAMY (kU/l), bispectral index, systolic blood pressure $(\mathrm{mmHg})$, and pulse rate $(/ \mathrm{min})$ are all indicated in the lower panel 


\section{Discussion}

ESD has been assumed to be a less invasive treatment, which is associated with reduced psychological and/or physiological stress experienced by the patient, compared with that in open surgery. However, the results of the present study clearly demonstrate, based on changes in the sAMY, that patients undergoing ESDs with DS do suffer from some stress in comparison to those patients who are treated under GA. To our knowledge, this is the first application of sAMY monitoring to objectively assess the stress experienced by patients during an ESD.

The production of salivary amylase is controlled by epinephrine secreted from the adrenal medulla, caused by enhanced activity of the sympathetic nervous-adrenomedullary system $[10,11]$. The sAMY has recently been shown to be a marker of sympathetic system activity $[6,7,10,12-14]$. When sudden and stressful stimuli are applied to the human body, the sympathetic fibers trigger the salivary gland, which secretes amylase before the gland responds to norepinephrine from the adrenal medulla; this is thought to be a faster response than the response to norepinephrine, and it usually occurs within minutes [15]. Cortisol is also suitable as a similar stress marker [16]. However, because the cortisol response is hormonally mediated, its reaction tends to be sluggish. Taking these characteristics into consideration, sAMY is therefore considered to be a real-time stress marker. Recently, a portable clinical instrument has been developed and marketed as a noninvasive, easy-to-use, and speedy sAMY measuring system [8]. Chromogranin A has also been used to assess the psychological stress caused during endoscopy [17]. However, this method uses an enzyme immunoassay and requires more time than the measurement of sAMY. Because measuring sAMY takes only $1 \mathrm{~min}$ after saliva sampling, it is very practical for assessing patients' stress level during endoscopy.

According to the results of the GA group in the present study; namely, that sAMY was maintained at a more strongly decreased level during the procedure than that at rest, the sympathetic tone was therefore completely controlled from start to finish. GA is preferred for ESDs requiring an extended time because GA can provide strict whole-body control [3]. The present results confirm this from the viewpoint of stress assessed by sAMY. In contrast to the GA group, the DS group showed an increase in SAMY just after the beginning of ESD. In spite of the administration of additional sedative agents, such as midazolam, appropriately, sAMY remained high and unstable. However, no significant difference was observed in sAMY between the GA and DS groups at the time of only sedation or anesthesia, before the beginning of ESD. Therefore, these results confirmed that the changes in SAMY in patients undergoing ESD did not occur simply owing to the different anesthesia methods.

The BIS Monitor is a device for estimating anesthetic depth from brain waves. A bispectral index of close to 100 indicates higher consciousness, and greater anesthetic depth is indicated when the index gets closer to $0[18,19]$. Naturally, regarding the anesthetic depth expressed by the bispectral index, a difference was observed between the two groups in the present study. However, according to the questionnaire results, the majority of the patients in the DS group, including the patient whose details are shown in Fig. 5, did not wake up or experienced no stress. Therefore, it is possible that the sAMY may not assess the level of consciousness, but instead that it monitors some other factor. Furthermore, the observation that pentazocine, but not midazolam, effectively reduced the high sAMY level as well as the body motion indicates that the stress shown by sAMY in a patient under DS is physiological stress; namely, excitation of the sympathetic nervous system caused by pain stimuli, rather than psychological stress. The sAMY has previously been reported to be useful for assessing pain in patients suffering from chronic low back or leg pain [20]. However, that report showed the sAMY to be related to the self-assessment of pain in a patient who was aware. Therefore, the present report is considered to be the first objective assessment of the analgesic level in patients who are unconscious.

The guidelines for the Sedation and monitoring of patients undergoing gastrointestinal endoscopic procedures [21] announced in 1995 by the American Society for Gastrointestinal Endoscopy clearly state that "It is the responsibility of every practitioner engaged in the performance of endoscopic procedures to maximize the benefit and safety to the patient." The advantage of these guidelines is that, besides offering definitions of the diagnosis and treatment, the guidelines suggest that patients can have painless and comfortable endoscopy. Until now, the degree of analgesia in patients undergoing ESD has not been fully understood. However, our discovery that the analgesic level can be easily monitored using SAMY may therefore positively contribute to the performance of safe and secure ESDs under DS.

In conclusion, GA for ESD inhibits sympathetic agitation in response to the stress of pain that is measured by SAMY to a much greater extent than DS, without exception. However, DS is also useful for ESD, irrespective of the operation time, as shown by the finding that most patients in the present study underwent nonstressful ESD under DS. ESD procedures can be performed safely under DS by assessing the analgesic level through sAMY and the sedative level using a BIS Monitor. In particular, sAMY is useful for decisions on the appropriate timing of administering analgesic agents. 
Acknowledgments This work was supported in part by a Grant-in-Aid from the Japanese Foundation for Research and Promotion of Endoscopy (JFE).

\section{References}

1. Gotoda T. Endoscopic resection of early gastric cancer. Gastric Cancer 2007;10:1-11.

2. Miyazaki S, Gunji Y, Aoki T, Nakajima K, Nabeya Y, Hayashi H, et al. High en bloc resection rate achieved by endoscopic mucosal resection with IT knife for early gastric cancer. Hepatogastroenterology 2005;52:954-8.

3. Naruse M, Inatsuchi S. Risk management in endoscopic submucosal dissection in upper gastrointestinal endoscopy: risk management for sedation in endoscopic submucosal dissection. Dig Endosc 2007;19:S2-S4.

4. Choi IJ, Kim CG, Chang HJ, Kim SG, Kook MC, Bae JM. The learning curve for EMR with circumferential mucosal incision in treating intramucosal gastric neoplasm. Gastrointest Endosc 2005;62:860-5.

5. American Society of Anesthesiologists Task Force on Sedation and Analgesia by Non-Anesthesiologists. Practice guidelines for sedation and analgesia by nonanesthesiologists. Anesthesiology 2002;96:1004-17.

6. Takai N, Yamaguchi M, Aragaki T, Eto K, Uchihashi K, Nishikawa Y. Effect of psychological stress on the salivary cortisol and amylase levels in healthy young adults. Arch Oral Biol 2004;49: 963-8.

7. Noto Y, Sato T, Kudo M, Kurata K, Hirota K. The relationship between salivary biomarkers and state-trait anxiety inventory score under mental arithmetic stress: a pilot study. Anesth Analg 2005;101:1873-6.

8. Yamaguchi M, Kanemori T, Kanemaru M, Takai N, Mizuno Y, Yoshida H. Performance evaluation of salivary amylase activity monitor. Biosens Bioelectron 2004;20:491-7.

9. Yamaguchi M, Deguchi M, Wakasugi J, Ono S, Takai N, Higashi T, et al. Hand-held monitor of sympathetic nervous system using salivary amylase activity and its validation by driver fatigue assessment. Biosens Bioelectron 2006;21:1007-14.
10. Chatterton RT Jr, Vogelsong KM, Lu YC, Ellman AB, Hudgens GA. Salivary alpha-amylase as a measure of endogenous adrenergic activity. Clin Physiol 1996;16:433-48.

11. Speirs RL, Herring J, Cooper WD, Hardy CC, Hind CRK. The influence of sympathetic activity and isoprenaline on the secretion of amylase from the human parotid gland. Arch Oral Biol 1974; $19: 747-52$.

12. van Stegeren A, Rohleder N, Everaerd W, Wolf OT. Salivary alpha amylase as marker for adrenergic activity during stress: effect of beta blockade. Psychoneuroendocrinology 2006;31:137-41.

13. Rohleder N, Nater UM, Wolf LM, Ehlert U, Kirschbaum C. Psychosocial stress-induced activation of salivary alpha-amylase: an indicator of sympathetic activity? Ann N Y Acad Sci 2004;1032: 258-63.

14. Nater UM, Marca RL, Florin L, Moses A, Langhans W, Koller $\mathrm{MM}$, et al. Stress-induced changes in human salivary alphaamylase activity - associations with adrenergic activity. Psychoneuroendocrinology 2006;31:49-58.

15. Skosnik PD, Chatterton RT Jr, Swisher T, Park S. Modulation of attentional inhibition by norepinephrine and cortisol after psychological stress. Int J Psychophysiol 2000;36:59-68.

16. Kirschbaum C, Hellhammer DH. Salivary cortisol in psychobiological research: an overview. Neuropsychobiology 1989;22: 150-69.

17. Fujimoto S, Nomura M, Niki M, Motoba H, Ieishi K, Mori T, et al. Evaluation of stress reactions during upper gastrointestinal endoscopy in elderly patients: assessment of mental stress using chromogranin A. J Med Invest 2007;54:140-5.

18. Glass PS, Bloom M, Kearse L, Rosow C, Sebel P, Manberg P. Bispectral analysis measures sedation and memory effects of propofol, midazolam, isoflurane, and alfentanil in healthy volunteers. Anesthesiology 1997;86:836-47.

19. Katoh T, Suzuki A, Ikeda K. Electroencephalographic derivatives as a tool for predicting the depth of sedation and anesthesia induced by sevoflurane. Anesthesiology 1998;88:642-50.

20. Shirasaki S, Fujii H, Takahashi M, Sato T, Ebina M, Noto Y, et al. Correlation between salivary alpha-amylase activity and pain scale in patients with chronic pain. Reg Anesth Pain Med 2007;32:120-3.

21. Sedation and monitoring of patients undergoing gastrointestinal endoscopic procedures. American Society for Gastrointestinal Endoscopy. Gastrointest Endosc 1995;42:626-9. 